\title{
PERCEPÇÃO AMBIENTAL SOBRE FLORESTAS URBANAS COMO FERRAMENTA DE PLANEJAMENTO E GESTÃO URBANA PARA CIDADES SUSTENTÁVEIS
}

\author{
Charles Costa COELHO 1 \\ https://orcid.org/0000-0002-5594-5584 \\ Ana Carolina BOSCHETTI 1 \\ https://orcid.org/0000-0001-8557-2443 \\ Marcelo Diniz VITORINO ${ }^{2}$ \\ https://orcid.org/0000-0002-9537-6275 \\ Tatiele Anete Bergamo FENILLI ${ }^{2}$ \\ https://orcid.org/0000-0003-3474-5935
}

Resumo: As árvores de rua desempenham um papel crucial na busca por cidades mais verdes e resilientes, pois fornecem diversos serviços ecossistêmicos (SE), melhorando a qualidade do ar, armazenando carbono, proporcionando sombra e diminuindo o efeito de ilha de calor urbano. O objetivo do estudo foi avaliar o nível de conscientização dos moradores da cidade de São Joaquim (Santa Catarina), a respeito das florestas urbanas. Para avaliação da percepção foi empregado um questionário misto, do tipo survey, com elementos predefinidos. Ao todo foram aplicados 300 questionários. A maior concentração dos entrevistados corresponde ao sexo masculino com $50,7 \%$, contra $49,3 \%$ do sexo feminino. A questão investir em árvores na cidade é desperdício de dinheiro público, apresentou uma grande concentração nas respostas discordantes, sendo superior a $90 \%$. Esse valor demonstra que os entrevistados são favoráveis que o dinheiro público seja investido na arborização urbana, seja através de novos plantios ou ainda manutenção/manejo e monitoramento. Os entrevistados demonstraram conhecer diretamente e indiretamente os benefícios gerados pelas florestas urbanas. Assim, é necessário a criação de ações coletivas entre gestores públicos e comunidade, com proposito de subsidiar e fomentar a arborização urbana em São Joaquim.

Palavras Chave: Ilhas de calor. Serviço ecossistêmico. Monitoramento.

Abstract: Street trees play a crucial role in the search for greener and more resilient cities as they provide diverse ecosystem services (SE), improving air quality, storing carbon, providing shade and lessening the effect of urban heat island. The objective of the study was to evaluate the level of awareness of the residents of São Joaquim (Santa Catarina) about urban forests. Perception assessment was performed using a mixed survey questionnaire with predefined elements. In all, 300 questionnaires were applied. The highest concentration of respondents corresponds to males with $50.7 \%$, compared to $49.3 \%$ females. The issue of investing in trees in the city is a waste of public money, there was a large concentration on discordant responses, being over $90 \%$. This value shows that respondents are in favor of public money being invested in urban afforestation, either through new plantings such as maintenance/management and monitoring. Respondents know directly and indirectly the benefits generated by urban forests. Thus, it is necessary to create collective actions between public managers and the community, with the purpose of subsidizing and promoting urban tree planting in São Joaquim.

Key Words: Heat islands. Ecosystem service. Monitoring.

1 Discentes do Programa de Pós-Graduação em Engenharia Florestal - PPGEF. Universidade Regional de Blumenau (FURB), Blumenau, SC, Brasil. ccoelho.florestal@gmail.com.

${ }^{2}$ Docentes do Programa de Pós-Graduação em Engenharia Florestal - PPGEF. Universidade Regional de Blumenau (FURB), Blumenau, SC, Brasil.
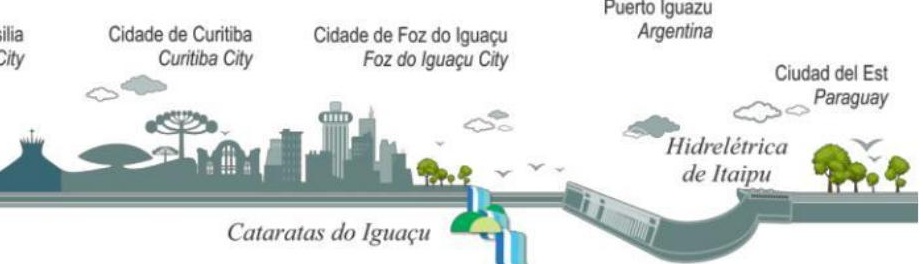


\section{International Journal of Environmental Resilience Research and Sriente (IJERRS)}

Revista Internacional Resiliência Ambiental Pesquisa e Ciência Saciedade 5.0 Resiliência Ambiental

\section{INTRODUÇÃO}

Nos últimos anos, uma ampla gama de pesquisas tem demonstrado que a presença de árvores nas cidades é obrigatória para minimizar os efeitos da densificação urbana sem precedente, que as cidades vêm apresentando (Sanesi e Chiarello, 2006; Soares et al., 2011; Gerstenberg e Hofmann, 2016). A atual situação exige cidades mais verdes, onde as árvores são elementos chave e importantes instrumentos no planejamento urbano.

As árvores de rua desempenham um papel crucial na busca por cidades mais verdes e resilientes, pois fornecem diversos serviços ecossistêmicos (SE), tal como, aumentar a habitabilidade do ambiente urbano reduzindo o escoamento de águas pluviais, melhorando a qualidade do ar, armazenando carbono, proporcionando sombra e diminuindo o efeito de ilha de calor urbana (Mullaney et al., 2015). Além disso, seu arranjo linear permite a conexão entre espaços verdes, contribuindo para uma Infraestrutura Verde Urbana (IVU) mais coesiva (Fernandes et al., 2015).

Cada avaliação feita para esses SE deve ser iniciada com uma abordagem social para considerar as percepções das partes locais interessadas (Cuni-Sanchez et al., 2016). Entender as preferências públicas em relação à biodiversidade, bens e serviços ecossistêmicos é importante para o manejo dos ecossistemas, já que a implementação e efetividade das intervenções de manejo frequentemente dependem do apoio da sociedade (Hirsch et al., 2011; Mace, 2014; Martín-López e Montes, 2015).

Além disso, as metodologias sociais para avaliar a percepção da comunidade em relação a SE são, atualmente, discrepantes (Felipe-Lucia et al., 2015; Menzel e Teng, 2009) e as abordagens padrão precisam ser desenvolvidas. Desse modo, o trabalho buscou avaliar o nível de conscientização dos moradores de São Joaquim a respeito das florestas urbanas. 


\section{International Journal of Environmental Resilience Research and Sriente (IJERRS)}

Revista Internacional Resiliência Ambiental Pesquisa e Ciência Saciedade 5.0 Resiliência Ambiental

\section{METODOLOGIA}

O estudo foi realizado no perímetro urbano da cidade de São Joaquim em Santa Catarina (Figura 01).

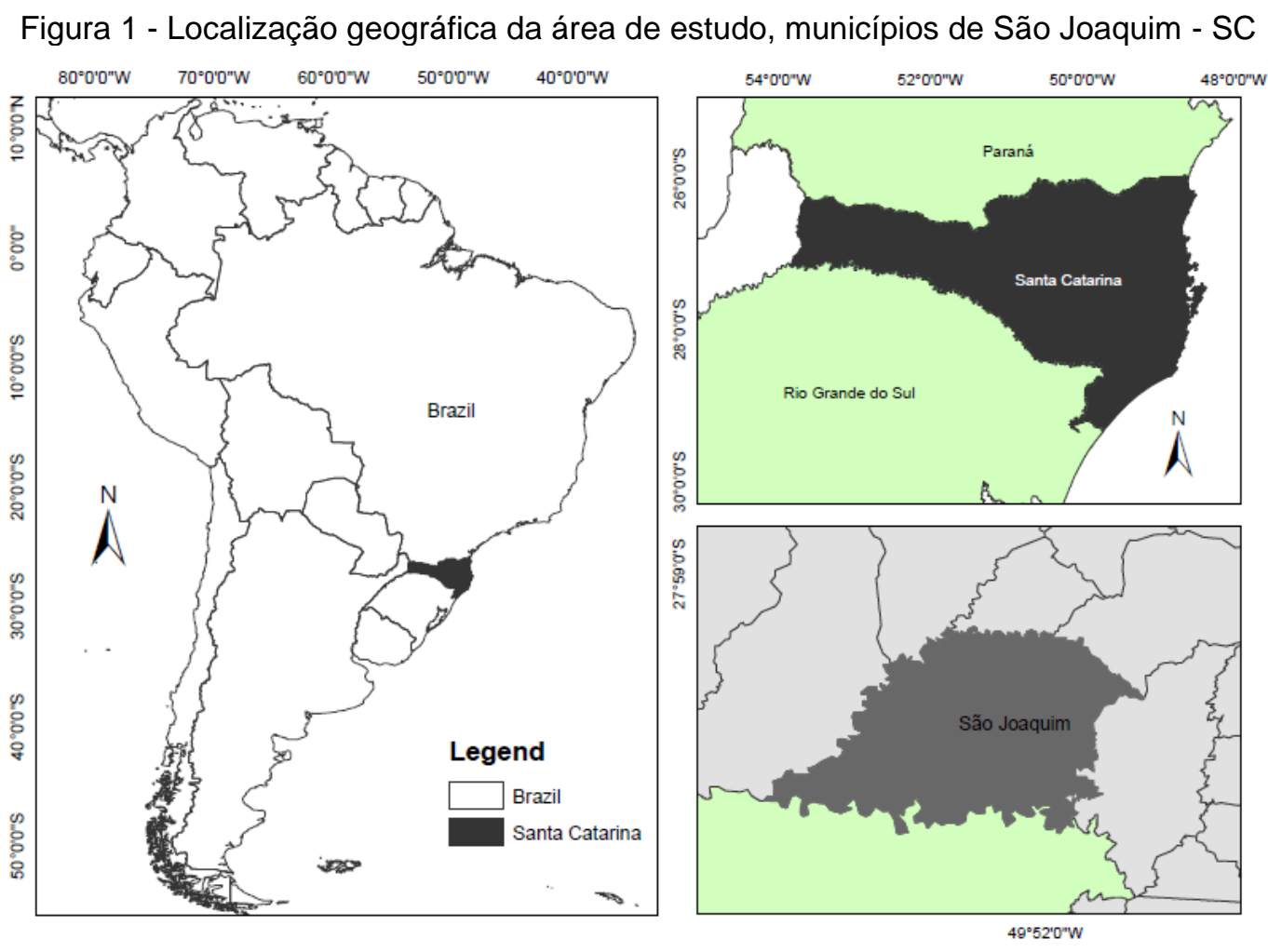

O município possui uma população de 24.812 habitantes (IBGE, 2019). A economia é voltada para agropecuária e fruticultura, tendo a produção de frutas de clima temperado como principal fonte de renda. A cada ano que passa, aumenta o número de pousadas, hotéis e restaurantes, devido ao crescente número de turistas que a cidade recebe no período do inverno, pois o município está entre as cidades mais frias do País (PREFEITURA MUNICIPAL DE SÃO JOAQUIM, 2019).

$A$ arborização presente atualmente em São Joaquim é resultado do que foi implantado logo nos primeiros anos, pelos primeiros moradores da cidade, sem 


\section{International Journal of Environmental Resilience Research and Srience (IJERRS)}

Revista Internacional Resiliência Ambiental Pesquisa e Ciência Saciedade 5.0 Resiliência Ambiental

qualquer tipo de planejamento. Com o passar dos anos, aliado ao crescimento urbano sem nenhum tipo de planejamento voltado para arborização urbana, as árvores acabaram sendo retiradas para dar espaço para construção civil, diminuindo ainda mais o número de indivíduos presentes na arborização urbana da cidade em estudo.

Para avaliação de percepção, foi empregado um questionário misto dividido em dois grupos, grupo das afirmações e grupo das perguntas (Figura 2), embasado na metodologia de Brun (2008), Viana (2013) e Coelho (2017).

Figura 2 - Estrutura do questionário de percepção aplicado no município de São Joaquim (SC)

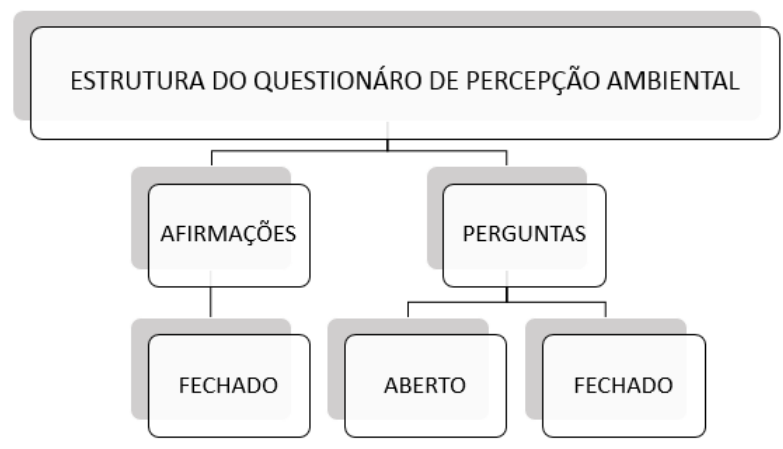

A pesquisa foi aplicada tipo survey. Ao todo foram aplicados 300 questionários. Nos surveys, independentemente do tamanho da população amostral a quantidade de 300 entrevistados apresenta um nível estatístico satisfatório de confiança, com erro amostral inferior a 10\% sobre a população real (ALRECK e SERRLE, 1995). A compilação dos dados foi realizada por meio de planilha Excel Microsoft $\AA$ e os dados foram submetidos a teste não paramétrico (Teste Qui-Quadrado X2), utilizando o Software BioEstat 5.3.

\section{RESULTADOS E DISCUSSÃO}

A maior concentração dos entrevistados corresponde ao sexo masculino com $50,7 \%$, contra $49,3 \%$ do sexo feminino (Tabela 1 ). Proporcionalmente a maior parte 


\section{International Journal of Environmental Resilience Research and Srience (IJERRS)}

Revista Internacional Resiliência Ambiental Pesquisa e Ciência Saciedade 5.0 Resiliência Ambiental

dos entrevistados encontra-se nas duas primeiras faixas etárias 18-24 (25,3\%), 25-34 (25\%) e na quarta faixa $45-54$ (15\%) e para o grau de escolaridade a faixa com maior proporção de entrevistado foi o Ensino Médio Completo (35,3\%).

Tabela 1 - Estatísticas demográficas dos entrevistados do estudo de percepção ambiental em São Joaquim - SC.

\begin{tabular}{|c|c|c|c|c|c|}
\hline & & \multicolumn{2}{|c|}{$\begin{array}{c}\text { Estatísticas demográficas } \\
\text { dos entrevistados }\end{array}$} & \multicolumn{2}{|c|}{$\begin{array}{c}\text { Estatísticas demográficas } \\
\text { reais }\end{array}$} \\
\hline & & Obs. (n.) & Freq. (\%) & Pop. (tot.) & Freq. (\%) \\
\hline \multirow{2}{*}{ Sexo } & Mas. & 152 & 50,7 & 12434 & 50,1 \\
\hline & Fem. & 148 & 49,3 & 12378 & 49,9 \\
\hline \multirow{6}{*}{ Faixa etária } & $18-24$ & 76 & 25,3 & - & - \\
\hline & $25-34$ & 75 & 25,0 & - & - \\
\hline & $35-44$ & 39 & 13,0 & - & - \\
\hline & $45-54$ & 45 & 15,0 & - & - \\
\hline & $55-65$ & 30 & 10,0 & - & - \\
\hline & $>65$ & 35 & 11,7 & - & - \\
\hline \multirow{7}{*}{$\begin{array}{l}\text { Grau de } \\
\text { Escolaridade }\end{array}$} & SE & 8 & 2,7 & - & - \\
\hline & EFC & 30 & 10,0 & - & - \\
\hline & $\mathrm{EFI}$ & 55 & 18,3 & - & - \\
\hline & EMC & 106 & 35,3 & - & - \\
\hline & EMI & 28 & 9,3 & - & - \\
\hline & ESC & 43 & 14,3 & - & - \\
\hline & ESI & 28 & 9,3 & - & - \\
\hline
\end{tabular}

Legenda: $\mathrm{SE}=$ Sem Escolaridade; $\mathrm{EFC}=$ Ensino Fundamental Completo; $\mathrm{EFI}=$ Ensino

Fundamental Incompleto; EMC = Ensino Médio Completo; EMI = Ensino Médio Incompleto; ESC = Ensino Superior Completo e ESI = Ensino Superior Incompleto

No grupo das afirmações (Figura 03), a maioria dos entrevistados disseram que discordam $(54 \%)$ e discorda plenamente $(27,33 \%)$ que as árvores não deveriam ser plantadas nas cidades e apenas $10 \%$ concordam com esta afirmação. Para a segunda afirmação tratando-se dos benefícios da arborização urbana, apontando que as árvores nas cidades deixam o local mais saudável e fresco, a maioria reconhece sua importância. Somando as respostas "concordo" e "concordo plenamente" a 


\section{International Journal of Environmental Resilience Research and Srience (IJERRS)}

Revista Internacional Resiliência Ambiental Pesquisa e Ciência Saciedade 5.૫ Resiliência Ambiental

porcentagem foi superior a $99 \%$. Dados parecidos foram encontrados por Viana (2013), com 70\% dos entrevistados reconhecendo os atributos benéficos da arborização.

Figura 3 -Proporção (\%) dada por afirmações do estudo de percepção ambiental sobre florestas urbanas, empregada no município de São Joaquim - SC

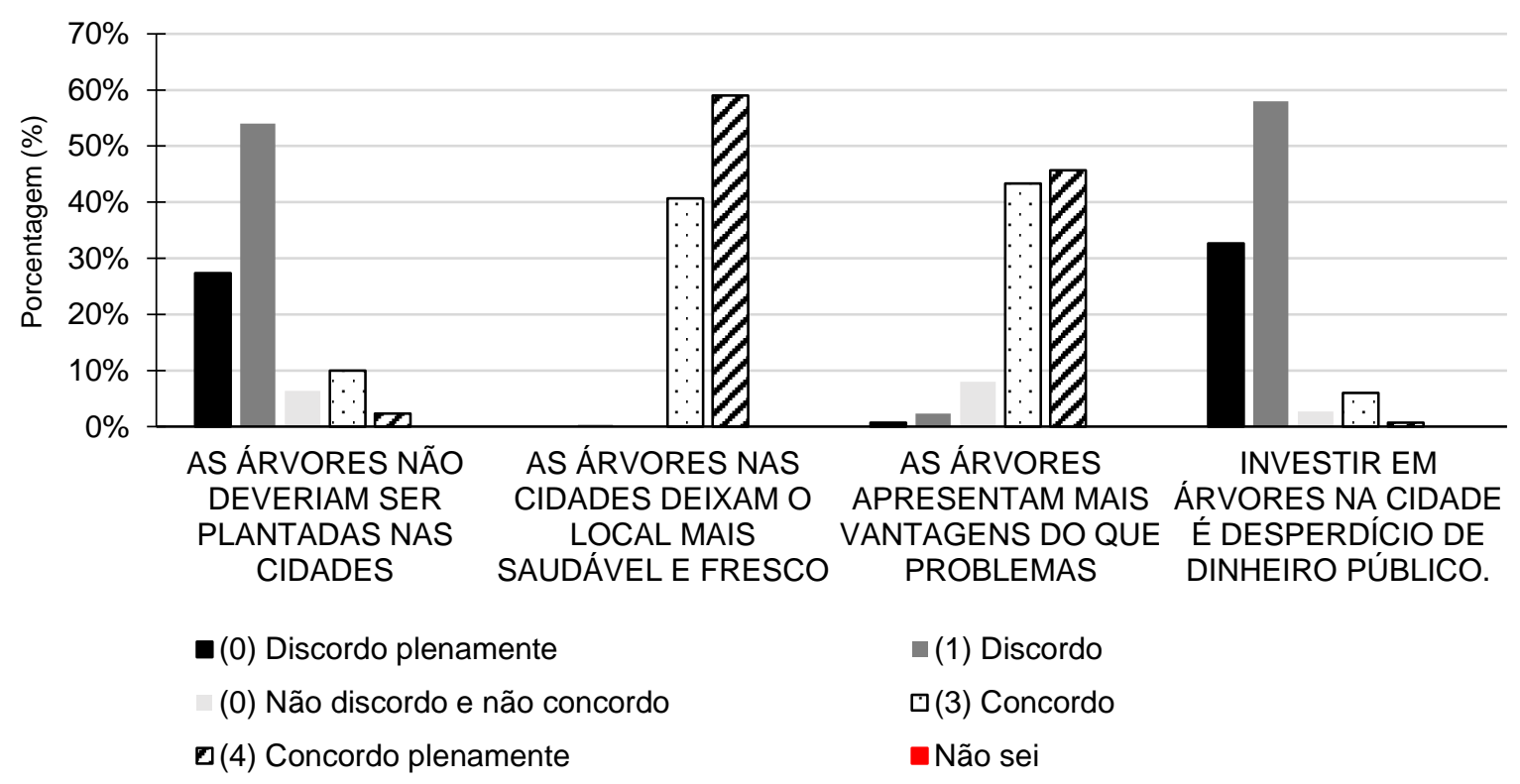

Na terceira afirmação (Figura 03 as árvores apresentam mais vantagens do que problemas, a maioria dos entrevistados reconhece o papel das florestas urbanas, sendo que $43,33 \%$ dos entrevistados optou pela resposta "concordo" e $45,67 \%$ pela resposta "concordo plenamente". Nas opiniões discordantes e "não discordo e não concordo" a soma representa $11 \%$, e os entrevistados apontam que um dos principais problemas é a sujeira causada pelas folhas.

É interessante observar que a afirmação, "investir em árvores na cidade é desperdício de dinheiro público", possui uma grande concentração nas respostas discordantes, sendo superior a $90 \%$. Esse valor demostra que os entrevistados são favoráveis que o dinheiro público seja investido na arborização urbana, seja através de novos plantios, ou como manutenção/manejo e monitoramento. Por outro lado, 


\section{International Journal of Environmental}

\section{Resilience Research and Science (IJERRS)}

Revista Internacional Resiliência Ambiental Pesquisa e Ciência Saciedade 5.0 Resiliência Ambiental

apenas 2 entrevistados responderam que "concordam plenamente" correspondendo a $0,67 \%$ do total da população amostral. Essa mesma população, demostrou-se desconfiada em relação ao destino do dinheiro público para serviços de arboricultura, além de serem mencionados constantes reclamações sobre os gestores públicos, em relação aos trabalhos desenvolvidos com as árvores urbanas na cidade.

No grupo das perguntas (Tabela 02), no sexo masculino $(n=121)$ e no sexo feminino ( $n=120)$ ambos acreditam que arborização urbana pode atrair turistas para a cidade.

Tabela 2 - Frequência e teste de significância para o grupo de perguntas, para o estudo de percepção ambiental em São Joaquim - SC.

\begin{tabular}{|c|c|c|c|c|c|c|c|}
\hline \multirow{2}{*}{\multicolumn{3}{|c|}{ QUESTIONÁRIO }} & \multicolumn{2}{|c|}{ N. Observado } & \multicolumn{3}{|c|}{$x^{2}$ teste } \\
\hline & & & Fem. & Mas. & $\mathrm{x}^{2}$ valor & GL & p-valor \\
\hline \multirow{2}{*}{\multicolumn{2}{|c|}{$\begin{array}{l}\text { Em seu ponto de vista você } \\
\text { acredita que arborização } \\
\text { urbana pode atrair turistas } \\
\text { para a cidade? }\end{array}$}} & Sim & 120 & 121 & 0.14 & 1 & 0.815 \\
\hline & & Não & 31 & 28 & - & - & - \\
\hline \multirow{5}{*}{$\begin{array}{l}\text { Você ajudaria com uma } \\
\text { contribuição financeira para } \\
\text { manutenção das árvores da } \\
\text { sua cidade? }\end{array}$} & & Sim & 123 & 110 & 2.58 & 1 & 0.148 \\
\hline & & Não & 28 & 39 & - & - & - \\
\hline & & $\mathrm{R} \$ 5,00 /$ ano & 32 & 20 & 1.89 & 2 & 0.388 \\
\hline & $\begin{array}{l}\text { se } \\
\text { sim: }\end{array}$ & 10,00/ano & 26 & 23 & - & - & - \\
\hline & & $>\mathrm{R} \$ 10,00 /$ ano & 21 & 23 & - & - & - \\
\hline
\end{tabular}

Legenda: $X^{2}=$ Símbolo do teste de Qui-quadrado; $G L=$ Grau de liberdade; $p=$ Nível de significância do teste

Os entrevistados apontam fatores como parques urbanos, túnel verde com arborização viária e principalmente a utilização de espécies nativas endêmicas da região para arborização, o qual influencia diretamente no turismo e aumento na economia da cidade. Quando aplicado o teste qui-quadrado $X^{2}$ para verificação entre a variável resposta (Sim/Não) e o sexo (Mas./Fem.), não houve diferença significativa $\left(X^{2}=0.14\right.$ e $\left.p=0.815\right)$, ou seja, não há associação entre as variáveis.

Quando questionado se o entrevistado ajudaria com uma contribuição financeira para a manutenção das árvores na cidade, o sexo feminino correspondeu as maiores afirmações, sendo 123 observações para resposta "Sim" e 28 observações para resposta "Não". Não houve diferença significativa entre os resultados $\left(X^{2}=2.58\right.$ e

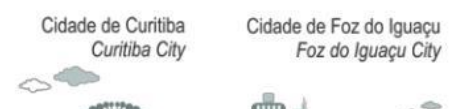




\section{International Journal of Environmental}

\section{Resilience Research and Sriente (IJERRS)}

Revista Internacional Resiliência Ambiental Pesquisa e Ciência Saciedade 5.0 Resiliência Ambiental

$\mathrm{p}=0.148$ ). Com objetivo de quantificar monetariamente o valor da contribuição para os cuidados com as árvores urbanas, os entrevistados que responderam "Sim" receberam uma escala de valor, para informar até quanto estava disponível em ajudar. Desse modo observou-se que a maior faixa foi de $R \$ 5,00 /$ ano, respectivamente 52 entrevistados, seguida pela faixa de $R \$ 5,00$ a 10,00 /ano e pela faixa de $R \$$ $>10,00 / a n o$. Não houve associação entre o valor da contribuição monetária por gênero $\left(X^{2}=1.89\right.$ e $\left.p=0.388\right)$.

\section{CONSIDERAÇÕES FINAIS}

Através do estudo de percepção conclui-se que os entrevistados conhecem diretamente e indiretamente os benefícios gerados pelas florestas urbanas, bem como, a importância para cidades sustentáveis. Os entrevistados demonstraram-se dispostos a contribuir financeiramente para a manutenção e monitoramento dessas áreas, fazendo-se necessária a criação de ações coletivas entre gestores públicos e comunidade, com propósito de subsidiar e fomentar a arborização urbana.

\section{REFERÊNCIAS}

ALRECK, P. L.; SETTLE, R. B. The survy research handbook: guidelines and strategies for conducting a survy. 2 ed. New York: McGraw-hill,1995.470p.

BRUN, F. G. K. Percepção sobre a arborização urbana no Bairro Camobi, Santa Maria-RS: estudo de um caso. 2008. 74 f. Monografia (Especialização em Educação Ambiental), Universidade Federal de Santa Maria, Santa Maria, 2008.

CUNI-SANCHEZ, A.; PFEIFER, M.; MARCHANT, R.; BURGUESS, N. D. Ethnic and locational differences in ecosystem service values: Insights from the communities in forest islands in the desert. Ecosystem Services, v. 19, p. 42-50, 2016.

FAGGIONATO, S. Percepção ambiental. Disponível em: <http://educar.sc.usp.br/biologia/textos/m_a_txt4.html>. Acessado em 11 de março de 2019.

FELIPE-LUCIA, M. R.; COMÍN, F. A.; ESCALERA-REYES, J. A framework for the social valuation of ecosystem services. Ambio, v. 44, n. 4, p. 308-318, 2015. 


\section{International Journal of Environmental}

\section{Resilience Research and Sriente (IJERRS)}

Revista Internacional Resiliência Ambiental Pesquisa e Ciência Saciedade 5.0 Resiliência Ambiental

FERNANDES, C.; MOTA, M.; LUFINHA, I.; SILVA, I.; MARQUES, P. F. Ok, cities grow, but the trees too! Now what? In Niin, G., Mishra, H.S. (Eds.), Proceedings of the ECLAS Conference "Landscapes in Flux", Sep 20-23, Tartu, Estonia, pp. 289-298

GERSTENBERG, T.; HOFMANN, M. Perception and preference of trees: A psychological contribution to tree species selection in urban areas. Urban Forestry \& Urban Greening, v. 15, p. 103-111, 2016.

IBGE - INSTITUTO BRASILEIRO DE GEOGRAFIA E ESTATÍSTICA. São Joaquim - SC. Disponível em: <https://cidades.ibge.gov.br/brasil/sc/urubici/panorama>. Acesso em: 25 de fev. de 2019.

LACERDA, N. P. et al. Percepção dos residentes sobre a arborização da cidade de São José de Piranhas-PB. Revista da Sociedade Brasileira de Arborização Urbana, Piracicaba-SP, v. 5, n. 4, p. 81-95, 2010.

MENZEL, S.; TENG, J. Ecosystem services as a stakeholder-driven concept for conservation science. Conservation Biology, v. 24, n. 3, p. 907-909, 2010.

MULLANEY, J.; LUCKE, T.; TRUEMAN, S. J. A review of benefits and challenges in growing street trees in paved urban environments. Landscape and Urban Planning, v. 134, p. 157-166, 2015.

PREFEITURA MUNICIPAL DE SÃO JOAQUIM. Disponível em:< http://www.saojoaquim.sc.gov.br/>. Acesso em: 25 de fev. 2019.

SANESI, G.; CHIARELLO, F. Residents and urban green spaces: The case of Bari. Urban Forestry \& Urban Greening, v. 4, n. 3-4, p. 125-134, 2006.

SOARES, A. L.; REGO, F. C.; McPHERSON, E. G.; SIMPSON, J. R.; PEPER, P. J.; XIAO, Q. Benefits and costs of street trees in Lisbon, Portugal. Urban Forestry \& Urban Greening, v. 10, n. 2, p. 69-78, 2011.

VIANA, S. M. Percepção e quantificação das árvores na área urbana do município de São Carlos, SP. 2013. Tese (Doutorado em Recursos Florestais) - Escola Superior de Agricultura Luiz de Queiroz, Universidade de São Paulo, Piracicaba, 2013.

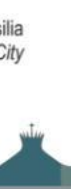

